\title{
Comprehensive Exploration for Proposing Hybrid Scheme upon Congestion Avoidance Algorithms
}

\author{
Wasai Shadab Ansari , ljaz Ali Shoukat, Atif M. AlAmri , Abdullah Al-Dhelaan, Mohsin \\ Iftikhar, Mudassar Ayub, Mohammad Serajuddin \\ College of Computer \& Information Sciences, King Saud University \\ P. O. Box. 51178, Riyadh 11543, Kingdom of Saudi Arabia (KSA).
}

\begin{abstract}
Congestion free services are ultimate preference of every network consumer and service providers. Variety of parameters like packet dropping rate, latency, jitter, throughput, bandwidth, fair response of resources, link utilization and queue length are responsible to fabricate or reduce congestion. Current TCP model for high speed networks is unstable and ineffectual due to slow response, large window size and fairness issues. The ideal and positive utilization of indicated factors can reduce congestion up to ideal strength with enhanced fairness. These entire factors cannot be handled with single congestion handling technique but a joint committee of congestion techniques can manage all these constraints. We considered packet loss as a primary congestion and fairness metric that differs with already conveyed hybrid congestion techniques that utilize delay as primary metric. We reviewed several congestion algorithms to find out most essential parameters to negate congestion in packet switched networks among the above mentioned parameters. We proposed a hybrid congestion handling technique after performing sufficient comparison with already conveyed hybrid congestion management techniques. Our propose hybrid congestion management technique (ECN + IFRC) is empirically superior to exiting hybrid congestion management techniques in some extents.
\end{abstract}

\section{General Terms}

Network Communication, Congestion and Flow Control, Networks Protocols, Network Services and measurements

\section{Keywords}

Congestion Avoidance, QoS, Latency, Jitter, Hybrid Congestion Schemes

\section{INTRODUCTION}

Most of applications require heavy contents with rapid transfer rate under the requirement of bulky bandwidth. In order to manage bandwidth and fair response there is need to manage congestion. The information regarding the congested situation collected by the sender may not be accurately reflecting the actual situation of congestion. This type of non-accurate information may caused worst situation because the network behaves like a black box for newly joined source (network node or machine), therefore in order to get fresh network situation the newly joined node initiates first request with small sending rate and increases the sending rate in next subsequent requests. The newly joined node may require the issuance of many requests in order get complete network situation. These types of network requests creates extra load on networks that may lead to congestion. The solution of this kind of congestion issue requires to judge the load on the behalf of hop counts and RTT rate ratio as discussed by Ijaz A. Shoukat and Iftikhar M. According to their opinions, network path is substantially amended with sufficient increment in hop counts and RTT values when congestion occurs [1]. Congestion management is reliant on four mutual algorithms (Efficient Retransmission, sluggish Start, Congestion Avoidance, Quick Recovery) and all these algorithms can be implemented under generic congestion handling protocols [2] : (1) Buffer based Congestion Protocol in which every node sends the packet to its downward node close to it if and only if the receiving node has some buffer capacity. (2) Rate Based Congestion Protocol - in which transmission rate is measured through both incoming and outgoing packet streams among the neighboring nodes by calculating the weight function. (3) Priority Based Congestion Protocol [3] - it deals with measuring hope's congestion severity (through packet arrival rate) and priority index of node (depending upon the weight of fairness).

Congestion investigation and control management can be done in three ways: (1) Detection, (2) Notification and (3) Adjustment of transmission rate [3]. Any control protocol that deals only with either index (delay) based indicator or loss based indicator cannot perform ideally against real time streaming video applications in a high speed network because for real time video streaming delays are not tolerated by users. Standard TCP mechanism and its several relatives like TCP New-Reno, TCP Illinois etc., are not sufficiently enough to deal with streaming application [4]. Our concern is with QoS of remote servers in network environment that mostly get engaged with congestion. We proposed a hybrid congestion handling technique that employs the ideal utilization of all congestion parameters to get enhanced result avoid congested situation.

\section{LITERATURE REVIEW}

The fixing of equalized throughput to each node does not mean ideal fairness. In 2006 the authors of study [3] reported that congestion is necessarily based on performance that can be improved by reducing the packet loss rate and fairness (Faire throughput utilization on each node). Furthermore they claimed that fairness itself is dependent on scheduling of packets. In 2011, the authors of study [5] proposed linear Matrix Inequality (LMI) based approach to deal with congestion situation. They studied the congestion occurrence under delay and link capacity parameters and they claimed that there approach is able to enhance performance in closed loop environment. But there study is only limited to judge two congestion constraints (delay and link capacity) that are not enough to get enhanced congestion free performance.

An ideal Packet switched network relies on routing decision and congestion free linkage. Efficient path decision making is done under routing strategy and congestion is the greatest hurdle in efficiency of transmission of remote queries. Present design of IP (Internet Protocol) can resolve single path routing with sluggish time degree and TCP (Transmission Control Protocol) 
requires end to end packet delivery under the management of fixed transmission rate through congestion window size limits that is not an effective solution for getting enhanced results [6]. The congestion window limits actually causes the increment in Round Trip Time (RTT) that severely effects the transmission rate[6]. HTCP(Hamilton TCP) [7] works on adaptive back-off strategy to attain a better efficiency and improved the performance over responsiveness in high speed networks. But it can't tackle the congestion state because of the rapid increment/ decrement in their window sizes with high flow traffic can results throughput degradation. BIC-TCP(Binary Increase Congestion TCP) [8] scheme was developed to overcome this drawback by executing a linear increment of window size at initial phase and then amplifying its value logarithmically towards the reference point. But this also undergoes the same RTT unfairness problem. According to the authors of study [9] the use of individual congestion control scheme cannot predict all type of congestion parameters to reflect actual loaded or congested situation. The authors of study [10] analyzed the joint effect of Binary Congestion Notification $(\mathrm{BCN})$ and Transport Control Protocol (TCP) with heterogeneous traffic that can trigger the performance by reducing multiple packet losses. Compound Transport Control Protocol (CTCP) [21] is a hybrid approach which includes a scalable delay based constituent into some loss based component like TCP Reno congestion avoidance scheme. When network path is underutilized, the delay based component increases sending rate rapidly and once the path utilized or bottleneck queue built, it automatically becomes nonaggressive i.e. it reduces the sending rate decently since delay based schemes have a feature of automatic adjustment of its aggressiveness on basis of link utilization[12] [13]. CTCP performs well in case of single network flow/link but perform poor when dealing with shared network links i.e. their performance measures degrade and poor fairness achieved [11].

Many other congestion handling schemes as well as hybrid congestion management schemes have already been reported as discussed in Table (1 and 2). Each congestion handling scheme either individual or hybrid has its own affirmative and feeble characteristics with a reality that these all schemes cannot handle all congestion parameters in ideal way. Therefore, there should be a hybrid approach through which all type of required congestion parameters can be judged in optimal way.

\section{COMPARISON OF CONGESTION TECHNIQUES}

We technically performed the comparison of several congestion handling algorithms in the presence of important congestion parameters to analyze their discrepancies and affirmative effects on QoS as discussed in Table 1.

Table 1: Comparison of Congestion Algorithms

AIMD (Additive Increase and Multiplicative Decrease) [14],[15],[16]

\begin{tabular}{|c|c|}
\hline Evaluation Factors & Detail and discussion \\
\hline Packet Dropping Probability (PDP) & Packets are lost when throughput rate becomes equal to buffer capacity. \\
\hline Latency & Latency will be large as the size of queue will be larger \\
\hline Jitter & $\begin{array}{l}\text { Dynamic increase and decrease causes jitter [17]. Its buffer size is quite large that can } \\
\text { create jitter situation too. <variable> }\end{array}$ \\
\hline Throughput & $\begin{array}{l}\text { It has the capability to decrease the buffer size additively to get optimal throughput. [18] } \\
\text { [19]. } \\
\text { Its throughput is dependent on its window size. <variable> }\end{array}$ \\
\hline Link/ Channel Capacity (Bandwidth) & $\begin{array}{l}\text { Channel capacity is severely affected due to its capability of multiplicative decrease. So it } \\
\text { is not a good solution for high bandwidth links. }\end{array}$ \\
\hline Fairness (Fair System Response) & $\begin{array}{l}\text { It has the capability to achieve fairness by allocating the resources in increasing and } \\
\text { decreasing fashion. [20]. }\end{array}$ \\
\hline Link utilization (achieved throughput ) & $\begin{array}{l}\text { High [18] [20] because it additively increases, so it is not an effective decision to use in } \\
\text { High Bandwidth Delay Product (HBDP) networks. }\end{array}$ \\
\hline Queue length & it uses round robin model to serve these flows. [17] \\
\hline \multicolumn{2}{|r|}{ RED(Random Early Detection)[21],[22],[23] } \\
\hline Evaluation Factors & Detail and discussion \\
\hline Packet Dropping Probability (PDP) & $\begin{array}{l}\text { As soon as queue size increases the packets loss occurs. } \\
\text { There is chance to drop packets when queue size grows enormously. [24] }\end{array}$ \\
\hline Latency & $\begin{array}{l}\text { Large buffer size causes latency as it is directly proportional to buffer delay [24]. } \\
\text { <variable> }\end{array}$ \\
\hline Jitter & - \\
\hline Throughput & Throughput is dependent on traffic load. 〈variable> \\
\hline Link/ Channel Capacity (Bandwidth) & It operates well with routers having high bandwidth capacity. [24] \\
\hline Fairness & $\begin{array}{l}\text { Fairness can be achieved by increasing the queuing delay since more bandwidth-delay } \\
\text { product is allotted for each flow and packet dropping for each flow reduces thereby } \\
\text { reducing the overall congestion. [25] }\end{array}$ \\
\hline Link utilization & $\begin{array}{l}\text { If the buffer size is small the link utilization is reduced drastically and the queuing delays } \\
\text { may get short [26] } \\
\text { Higher the queue occupancy value will be the better link utilization but it causes more } \\
\text { queuing delays. [24] <variable> }\end{array}$ \\
\hline Queue & Packets loss occurs on average increment in queue size. \\
\hline \multicolumn{2}{|r|}{ BCN(Backward Congestion Notification)[27], [28] } \\
\hline Evaluation Factors & Detail and discussion \\
\hline
\end{tabular}




\begin{tabular}{|c|c|}
\hline & $\begin{array}{l}\text { In normal network condition probability of dropping is nearly zero. [29] } \\
\text { Queue has a limit. When limit is overflowed packet loss occurs. <variable> }\end{array}$ \\
\hline Latency & $\begin{array}{l}\text { Latency is dependent on distance metric. } \\
\text { Latency is also dependent in retransmission [29]. 〈variable> }\end{array}$ \\
\hline Jitter & $? ? ?$ \\
\hline Throughput & (TCP-BCN) combination is better for good throughput. \\
\hline \multicolumn{2}{|l|}{ Link/ Channel Capacity (Bandwidth) } \\
\hline Fairness & $\begin{array}{l}\text { TCP-BCN combination is better for good Fairness. Unfairness is dependent on long } \\
\text { distance. }\end{array}$ \\
\hline Link utilization & TCP-BCN combination is better for getting optimal link utilization \\
\hline Queue & Queue has a limit. When limit is overflowed packet loss occurs. \\
\hline \multicolumn{2}{|r|}{ CPT(Choke Packet technique)[30] } \\
\hline Evaluation Factors & Detail and discussion \\
\hline Packet Dropping Probability (PDP) & If Avg. queue Size > Max. allocated-Value, Then packets begins to drop. \\
\hline Latency & $\begin{array}{l}\text { Latency depends on the processing delay requires for Choke condition. } \\
\text { If both CPT-UDP are combined in parallel then latency is short. <variable> }\end{array}$ \\
\hline Jitter & Jitter is more in case of CPT used with UDP. \\
\hline Throughput & $\begin{array}{l}\text { In case of TCP, it gives higher throughput. } \\
\text { In case of UDP, it gives minimum throughput. [31] }\end{array}$ \\
\hline Link/ Channel Capacity (Bandwidth) & Channel capacity is not fully utilized in this algorithm. [32] \\
\hline Fairness & \\
\hline Link utilization & Good in case of responsive sources. \\
\hline Queue & \\
\hline \multicolumn{2}{|r|}{ ECN(Explicit Congestion Notification) [33],[34] } \\
\hline Evaluation Factors & Detail and discussion \\
\hline Packet Dropping Probability (PDP) & $\begin{array}{l}\text { ECN is effective because it has low packet dropping rather to TCP. [33],[34] } \\
\text { ECN - RED also have low rate of packet dropping. [33],[34] } \\
\text { But in case of dealing with satellite networks packet get lost due to buffer overflow. [35] }\end{array}$ \\
\hline Latency & $\begin{array}{l}\text { ECN possesses low latency when it is combined with TCP. } \\
\text { But high latency occurs in case of satellite networks as these networks causes high } \\
\text { propagation delays resulting in late congestion notification. [35] }\end{array}$ \\
\hline Jitter & $\begin{array}{l}\text { ECN has low rate of jitter because it has lower packet lose rate which makes the } \\
\text { congestion window more consistent. [36]. }\end{array}$ \\
\hline Throughput & $\begin{array}{l}\text { Higher throughput can be achieved in case of small gateway buffer size values in } \\
\text { comparison to drop tail and RED algorithm but in opposite case it doesn't so. [36] }\end{array}$ \\
\hline Link/ Channel Capacity (Bandwidth) & ECN bandwidth allocation is very good. \\
\hline Fairness & Resources are utilized fairly in ECN as it has low packet lose ratio [36]. \\
\hline Link utilization & $\begin{array}{l}\text { ECN - TCP provides } 100 \% \text { link utilization even in presence of queuing delay and } \\
\text { propagation. }\end{array}$ \\
\hline Queue & Average size of queue is noted for getting the level of congestion. \\
\hline \multicolumn{2}{|r|}{ EDD(Earliest due Date) [37],[38],[39],[40] } \\
\hline Evaluation Factors & Detail and discussion \\
\hline Packet Dropping Probability (PDP) & $\begin{array}{l}\text { Packets may get drop when sum of local relative deadlines is more than the end to end } \\
\text { deadline of connection i.e., throughput suffers less when deadlines monitored carefully. }\end{array}$ \\
\hline Latency & Depends on network nodes. <variable> \\
\hline Jitter & It depends on the degree of traffic / huge traffic (bursty traffic). <variable > \\
\hline Throughput & Throughput is optimal. [40]. \\
\hline Link/ Channel Capacity (Bandwidth) & Fair among intermediate nodes and it utilizes the channel capacity fairly well.[38] \\
\hline Fairness & $\begin{array}{l}\text { Resources are fairly utilized in successful condition but unfair in case of un-successful } \\
\text { condition. [37]. }\end{array}$ \\
\hline Link utilization & - \\
\hline Queue & - \\
\hline \multicolumn{2}{|r|}{ CAB(Congestion Avoidance Bit) Scheme [41],[42] } \\
\hline Evaluation Factors & Detail and discussion \\
\hline Packet Dropping Probability (PDP) & Approximately zero. [42] \\
\hline Latency & Low latency in presence of round trip delay metric. It is a fast network protocol for users. \\
\hline Jitter & Dependent on the change of window size. [41] \\
\hline Throughput & Routers operate efficiently, when traffic operates below knee that results fair throughput. \\
\hline Link/ Channel Capacity (Bandwidth) & In optimal case, it is good. \\
\hline Fairness & $\begin{array}{l}\text { The binary bit is fairly allocated to all resources by using global optimality } \\
\text { concept.[FAIR] }\end{array}$ \\
\hline Link utilization & - \\
\hline Queue & Average queue size is used as a metric to decide the load condition. [42]. \\
\hline
\end{tabular}




\begin{tabular}{|c|c|}
\hline \multicolumn{2}{|r|}{ TFRCP(TCP Friendly Rate Control Protocol) } \\
\hline Evaluation Factors & Detail and discussion \\
\hline Packet Dropping Probability (PDP) & $\begin{array}{l}\text { Packet lose ratio is dependent on Round Trip Time (RTT) as much as RTT increases, the } \\
\text { packet lose will be more. [43]. <variable> }\end{array}$ \\
\hline Latency & Latency is dependent on RTT if RTT is low the latency will be lower [44]. <variable> \\
\hline Jitter & $\begin{array}{l}\text { Jitter is dependent on queuing delay and RTT, as more RTT or delay as will be the jitter. } \\
\text { [43]. <variable> }\end{array}$ \\
\hline Throughput & $\begin{array}{l}\text { Throughput is dependent on the quality of signals as high quality signal have more } \\
\text { throughput. [44]. <variable> }\end{array}$ \\
\hline Link/ Channel Capacity (Bandwidth) & Bandwidth / channel capacity is poorly utilized due to RTT delays. \\
\hline Fairness & $\begin{array}{l}\text { It is relatively fair to TCP because it re-computes its connection rate only after every } \\
\text { " re-computation" time unit. [FAIR] [44]. }\end{array}$ \\
\hline Link utilization & Not good. [45]. \\
\hline Queue & - \\
\hline \multicolumn{2}{|r|}{ XCP(Explicit Control Protocol) } \\
\hline Evaluation Factors & Detail and discussion \\
\hline Packet Dropping Probability (PDP) & Approximately zero due to good resources utilization. [46]. \\
\hline Latency & $\begin{array}{l}\text { The average feedback value for all the links exceeds } 0 \text {, link capacity will be fully shared } \\
\text { and the queuing delay (Latency) will be minimum i.e., nearly to zero.[47]. }\end{array}$ \\
\hline Jitter & Less as compared to TCP [46]. \\
\hline Throughput & $\begin{array}{l}\text { The performance or throughput may degrades in shared access media environment like } \\
\text { radio/satellite communication. [32].Throughput increases proportionally to the increase in } \\
\text { value of average feedback. [46] }\end{array}$ \\
\hline Link/ Channel Capacity (Bandwidth) & Fully optimized [46]. \\
\hline Fairness & It is good in terms of fairness compare to other (end to end schemes)TCP schemes [48]. \\
\hline Link utilization & $\begin{array}{l}\text { Good. [31]. Link utilization is full when average feedback value is greater than zero, and } \\
\text { this scheme works efficiently. [33] }\end{array}$ \\
\hline Queue & Queue length remains constant [48]. \\
\hline \multicolumn{2}{|r|}{ DCCP (Datagram Congestion Control Protocol) } \\
\hline Evaluation Factors & Detail and discussion \\
\hline Packet Dropping Probability (PDP) & $\begin{array}{l}\text { Packet dropping probability is non-zero because this scheme is intended for real-time } \\
\text { traffic data and prefers the timely delivery instead of reliable or in-order delivery of data. } \\
\text { [49] }\end{array}$ \\
\hline Latency & $\begin{array}{l}\text { Minimum because, This scheme focus on timeliness delivery of data and not required } \\
\text { reliability and timing constraint [49]. }\end{array}$ \\
\hline Jitter & Jitter is maximized as compare to UDP, especially in case of multiple hope counts [50]. \\
\hline Throughput & $\begin{array}{l}\text { Maximized because, This scheme focus on timeliness delivery of data and not required } \\
\text { reliability and timing constraint [49]. }\end{array}$ \\
\hline Link/ Channel Capacity (Bandwidth) & $\begin{array}{l}\text { This method is suitable for varying bandwidth/channel capacity because it consists of } \\
\text { various transport layer type protocols for its operation. [49] }\end{array}$ \\
\hline Fairness & Provide well fairness [51]. \\
\hline Link utilization & - \\
\hline Queue & $\begin{array}{l}\text { In this scheme data are not queued for final delivery to applications unlike TCP and other } \\
\text { protocols instead the queued data may go forward only after the accordance of featured } \\
\text { values and options. [52] }\end{array}$ \\
\hline \multicolumn{2}{|r|}{ RCP(Rate Control Protocol ) } \\
\hline Evaluation Factors & Detail and discussion \\
\hline Packet Dropping Probability (PDP) & $\begin{array}{l}\text { Packet losing depends upon the change of networking condition and RTT [53]. } \\
\text { <variable> }\end{array}$ \\
\hline Latency & Lower Latency as router offers uniformly flow with small delay. [52]. \\
\hline Jitter & Jitter only occurs when total flow is larger than link capacity [52]. \\
\hline Throughput & It gives better throughput even in multiple bottlenecks rather to TCP [53]. \\
\hline Link/ Channel Capacity (Bandwidth) & Fully utilized [54]. \\
\hline Fairness & Resource sharing is fair [54]. \\
\hline Link utilization & Links are fully utilized [53]. \\
\hline Queue & Length is quite large [55]. \\
\hline \multicolumn{2}{|r|}{ BPT(Back Pressure Technique) } \\
\hline Evaluation Factors & Detail and discussion \\
\hline Packet Dropping Probability (PDP) & $\begin{array}{l}\text { Packet dropping probability can be reduced by ADPCM(Adaptive differential Pulse code } \\
\text { Modulation technique) [56] }\end{array}$ \\
\hline Latency & $\begin{array}{l}\text { Large queues are maintained in ICN(Intermittent connected Network) so delays are } \\
\text { longer than normal and high latency achieved due to maximized rate controller utility and } \\
\text { operating close in proximity of capacity region. [57] }\end{array}$ \\
\hline Jitter & Propagation of backpressure is too slow causing the fluctuation in flows of packet since \\
\hline
\end{tabular}


network state changes frequently that caused jitter. [47]

\begin{tabular}{|c|c|}
\hline Throughput & Optimal [57] [58]. \\
\hline Link/ Channel Capacity (Bandwidth) & Network capacity is highly utilized. [57] [58]. \\
\hline Fairness & $\begin{array}{l}\text { There is a tradeoff between fairness and delays, fairness improved with the increasing } \\
\text { cost of latency otherwise fairness is not achieved. [58] <variable> }\end{array}$ \\
\hline Link utilization & $\begin{array}{l}\text { Links are fairly utilized as they are scheduled regularly and their rates are computed at } \\
\text { each time by controller. [58] }\end{array}$ \\
\hline Queue & $\begin{array}{l}\text { Large queues maintained only at intermittently connected nodes, rest nodes have small } \\
\text { queues [57]. }\end{array}$ \\
\hline \multicolumn{2}{|r|}{ IFRC(Interference aware fair rate control) Scheme } \\
\hline Evaluation Factors & Detail and discussion \\
\hline Packet Dropping Probability (PDP) & $\begin{array}{l}\text { Packet dropping probability is almost zero even having small buffer sizes because it's rate } \\
\text { adaptation quality is much effective. [59] }\end{array}$ \\
\hline Latency & $\begin{array}{l}\text { When IFRC is implemented for retransmission on link level, it uses software ACKS that } \\
\text { may cause delays and increase the latency thereby reducing throughput. [60] }\end{array}$ \\
\hline Jitter & $\begin{array}{l}\text { It is an adaptive rate control scheme which means, rate lies between a minimal difference, } \\
\text { hence less jitter is experienced in this scheme. [61] }\end{array}$ \\
\hline Throughput & $\begin{array}{l}\text { Overall, it gives a higher throughput for protocols that use link-quality metrics in order to } \\
\text { establish the flow routing tree otherwise it provides minimum throughput in order to } \\
\text { reduce number of dropped packets. [59] < variable> }\end{array}$ \\
\hline Link/ Channel Capacity (Bandwidth) & $\begin{array}{l}\text { Capacity is fairly utilized through all the nodes by estimating the transmission time for } \\
\text { packets [62]. }\end{array}$ \\
\hline Fairness & $\begin{array}{l}\text { It employs a distributed rate adaptation technique to achieve fairness and support } \\
\text { weighted fair allocation. [59] }\end{array}$ \\
\hline Link utilization & $\begin{array}{l}\text { Link utilization is maximized and buffer dropping rates is almost zero which is the } \\
\text { property of this scheme. [62][61] }\end{array}$ \\
\hline Queue & Average queue lengths are monitored to find out the emerging congestion [59]. \\
\hline \multicolumn{2}{|r|}{ PBS(Partial Buffer Sharing) Scheme } \\
\hline Evaluation Factors & Detail and discussion \\
\hline Packet Dropping Probability (PDP) & $\begin{array}{l}\text { Packet dropping probability decreases when threshold position increases but in case of } \\
\text { delay tolerant traffic it continuous to increase. [63] <variable> }\end{array}$ \\
\hline Latency & $\begin{array}{l}\text { Latency is high because, response time increases for both delay tolerant and delay } \\
\text { sensitive traffic streams when threshold position increases. [63] }\end{array}$ \\
\hline Jitter & $\begin{array}{l}\text { It employs space priorities for different multimedia traffic classes in order to avoid the } \\
\text { jitter [63]. }\end{array}$ \\
\hline Throughput & Throughput depends upon delays [64]. \\
\hline Link/ Channel Capacity (Bandwidth) & Buffers are highly utilized for high priority traffic data. [64] \\
\hline Fairness & $\begin{array}{l}\text { It uses a threshold value that limits the access to buffer space against each higher/lower } \\
\text { priority traffic, and hence it cannot achieve better fairness among the resources, but with } \\
\text { CBS (Complete buffer sharing), better fairness can be achieved. [65]. }\end{array}$ \\
\hline Link utilization & Links are also utilized fairly in case of large threshold values. [63]. \\
\hline Queue & $\begin{array}{l}\text { The average queue length plays an influential part for allocating the threshold positions. } \\
\text { [63]. }\end{array}$ \\
\hline \multicolumn{2}{|r|}{ CTCP(Compound TCP) } \\
\hline Evaluation Factors & Detail and discussion \\
\hline Packet Dropping Probability (PDP) & $\begin{array}{l}\text { Packet dropping is zero when dealing with single flow but in shared network, it loses the } \\
\text { packets. [13] <Average or variable> }\end{array}$ \\
\hline Latency & $\begin{array}{l}\text { Some network latency is experienced even dealing with single network flow but slightly } \\
\text { lower than CUBIC TCP.[13] <variable / average> }\end{array}$ \\
\hline Jitter & Jitter is minimized through the use of synergized approach. \\
\hline Throughput & $\begin{array}{l}\text { With single or shared network connection severe degradation in throughput is } \\
\text { observed.[66] [67]. }\end{array}$ \\
\hline Link/ Channel Capacity (Bandwidth) & Efficient due to scalable window management rule. [12] \\
\hline Fairness & Good in case of single link utilization but poor incase of shared link utilization. [11] \\
\hline Link utilization & $\begin{array}{l}\text { It achieves efficient link utilization by having a rapid increase rule in its delay based } \\
\text { components e.g. multiplicative increase. [12] }\end{array}$ \\
\hline Queue & $\begin{array}{l}\text { Queue sizes are relatively shorter since it uses delay based approach as primal index of } \\
\text { congestion and hence window size is degraded before the happening of congestion.[13] }\end{array}$ \\
\hline
\end{tabular}

To get optimized congestion free situation the throughput and link utilization should be maximized. Furthermore, the queue length should be ideal, packet loss ratio and latency should be considerably minimized. The implementation of single congestion handling approach may not get the actual values of all parameters. 


\section{DISCUSSION AND ANALYSIS}

Web traffic is progressively growing; the size of indexed web contents was estimated more than 25 billion of web pages in January 2011 by the authors of study [70]. Growing volume of web is causing bulky network traffics. Complex network architecture and large number of internet traffic creating extra load on communication networks. In loaded situation, packet dropping is occurred at router's end that causes substantial increment in round trip times as well as in hop counts and this situation is designated as congestion [1]. Happening of latency in any remote service is caused by large incremental variations in round trip times because latency itself is the sum of all packet's round trip delays [71], therefore handling of load and congestion are the backbone to acquire quality oriented remote services in communication networks. Several hybrid congestion control schemes have been proposed that utilize the delay as primary indicator of congestion and packet loss as secondary indicator to control congestion. our analysis differs with the selection of delay as a primary metric and support to select packet loss as an essential metric to get optimal results. We summarized our results in Table 2 on the behalf of previously discussed comparison of congestion techniques in Table 1 . We combined the two congestion techniques $(\mathrm{ECN}+\mathrm{IFRC})$ in order to satisfy most important constraints (Packet dropping, link utilization) because the ideal satisfaction of other remaining congestion constraints is dependent on the satisfaction of these two constraints. We compared our proposed hybrid congestion management technique (ECN +IFRC) with prior hybrid congestion techniques (CPT-UDP [9] , TCP-BCN [8], C-TCP [21] , ECN - TCP [10] [11] , ECN - RED [10] [11] ) as summarized in Table 2.

Table 2 : Comparison results of Hybrid Congestion Techniques

\begin{tabular}{|c|c|c|c|c|c|c|c|c|}
\hline $\begin{array}{l}\text { Hybrid } \\
\text { Techniques }\end{array}$ & $\begin{array}{l}\text { Packet Dropping } \\
\text { Probability (PDP) }\end{array}$ & Latency & Jitter & Throughput & $\begin{array}{l}\text { Link/ Channel } \\
\text { Capacity } \\
\text { (Bandwidth) }\end{array}$ & \begin{tabular}{|l} 
Fairness \\
(Fair System \\
Response)
\end{tabular} & $\begin{array}{l}\text { Link } \\
\text { Utilization }\end{array}$ & Queue length \\
\hline CPT-UDP & Variable & Short & High & Minimum & Fully utilized & $? ? ?$ & Good & $? ? ?$ \\
\hline TCP-BCN & Variable & Variable & $? ? ?$ & Good & $? ? ?$ & Good & Optimal & Define Limits \\
\hline C-TCP & $\begin{array}{l}\text { Average or } \\
\text { variable }\end{array}$ & $\begin{array}{l}\text { variable OR } \\
\text { average }\end{array}$ & Min. & $\begin{array}{l}\text { severe } \\
\text { degradation }\end{array}$ & efficient & Good & Efficient & Shorter \\
\hline $\mathrm{ECN}-\mathrm{TCP}$ & \begin{tabular}{|l} 
Effective \\
( it means not low \\
and not zero)
\end{tabular} & Low & Low & High & Very Good & Fair & $100 \%$ & Average \\
\hline ECN - RED & Low & Low & Low & High & Very Good & Fair & Variable & Average \\
\hline $\begin{array}{l}\text { ECN + IFRC } \\
\text { Proposed }\end{array}$ & Zero & Low & Low & High & Good & Fair & Maximum & Average \\
\hline
\end{tabular}

According to our analysis, Packet dropping is the key metric rather to select delay as primary metric. Our suggested primary metric (packet loss) play a vital role in congestion occurrence because when packet starts to drop then it means there is a measureable latency (end to end delay) with jitter, limited throughput, poor bandwidth, unfair node response, limited link utilization and large queue length. Hence, this realistic trait clearly invokes that in suggesting hybrid congestion handling technique, packet dropping probability should be equivalent to zero as in the case of our suggested hybrid congestion technique $(\mathrm{ECN}+\mathrm{IFRC})$. In case of previously proposed hybrid congestion technique (ECN + TCP) packet dropping is effective (non-zero) and link utilization is $100 \%$ therefore; it possesses the more chances of congestion rather to our suggested hybrid congestion handling technique (ECN + IFRC). Furthermore, the other prior hybrid technique (ECN + RED) cannot provide better link utilization and packet dropping capability as compared to our proposed scheme. ECN-RED has low packet dropping rate (Non-Zero) and link utilization is ambiguous because it depends on the buffer size and if buffer size is small the link utilization is poor and if buffer is fully occupied then it increases the delay. Hence, proposed hybrid congestion scheme $(\mathrm{ECN}+\mathrm{IFRC})$ is superior to prior schemes.

\section{CONCLUSION}

Packet dropping is a prime metric rather to delay metric because the ideal control of packet loss ratio means ideal utilization of delay. Congestion escaping can enormously be improved with a joint committee of congestion handling schemes having packet dropping rate equivalent to zero with maximum link utilization because these ideal values mean all other congestion parameters (Latency, Jitter, Throughput, bandwidth, Fairness and Queue length) are ideally be satisfied. Minimized or equivalent to zero percent packet dropping rate lies under maximum link utilization and these both parameters are further dependent on all other congestion parameters. Our proposed hybrid congestion handling scheme [Explicit Control Notification $(\mathrm{ECN})+$ Interference aware fair rate control (IFRC)] possesses equivalent to zero percent packet dropping rate with maximum link utilization as compared to prior conveyed hybrid congestion management schemes (ECN-RED, ECN-TCP). Hence, our convey approach is superior to the existed congestion management schemes in terms of all discussed congestion parameters, therefore, we confidently advised the researchers to utilize our proposed congestion managing scheme to avoid congestion in packet switched networks.

\section{REFERENCES}

[1] Shoukat I.A., Iftikhar M. 2011. An experimental performance evaluation of different remote servers to analyze the effect of divergent load and congestion, International Journal of the Physical Sciences, Vol. 614. pp. 3354-3368, 18 July, 2011. 
[2] Sheu JP, Chang L. et al. 2009. Hybrid Congestion Control Protocol in Wireless Sensor Networks, Journal of Information Science And Engineering 25, 1103-1119 2009).

[3] Wang C., Sohraby K., Lawrence V. et al. 2006. Prioritybased congestion control in wireless sensor networks, in Proc. of the IEEE International Conference on Sensor Networks, Ubiquitous, and Trustworthy Computing, 2006, pp. 22-31.

[4] Floyd S., Handley M., Padhye J. et al. 2000. Equationbased congestion control for UNICAST applications, in Proceedings of ACM SIGCOMM 2000, pp. 43-56.

[5] Barzamini R., Shafiee M. 2011. LMI Based Switching Congestion Controller for Multiple Bottleneck Packet Switching Networks, Journal of American Science, Vol. 76. PP 254-261).

[6] Cominetti R., Guzman C. 2011. Network Congestion Control with Markovian Multipath Routing, in Proc. International conference on NETwork Games, COntrol and Optimization NetGCOOP 2011).

[7] Leith D., Shorten R.2004. H-TCP: TCP for high-speed and long-distance networks, PFLDnet, 04, in Meeting IETF63, Paris.

[8] Xu L., Harfoush K., Rhee I.2004. Binary increase congestion control BIC) for fast long-distance networks, 4 : PP2514-2524) IEEE.

[9] Ahmad S., Mustafa A., Ahmad B. et al. 2009. Comparative Study Of Congestion Control Techniques In High Speed Networks, International Journal of Computer Science and Information Security, Vol.6, No. 2, 2009.

[10] Sisalem D., Schulzrinne H. 1996. Congestion Control in TCP: Performance of Binary Congestion Notification Enhanced TCP Compared to Reno and Tahoe TCP, Commission of European Communities CEC) under project R2116 TOMQAT, IEEE

[11] Hayato Itsumi, Miki Yamamoto 2010. Improving Fairness between CUBIC and Compound TCP, IEICE Technical Report NS2010-160, pp. 103-108, 2010 in Japanese).

[12] Tan K., Song J., Zhang Q., Sridharan M. 2006. A Compound TCP Approach for High-speed and Long Distance Networks, Infocom 2006, 25th IEEE Communications Society, pp. 1-12

[13] Oura R., Yamaguchi S. 2011. Fairness Analysis among Modern TCP Congestion Avoidance Algorithms using Actual TCP Implementation and Actual Network Equipments, 2nd Intl Conference on networking and Computing, IEEE, pp. 297-299

[14] Chiu D.M., Jain R. 1998. Analysis of the Increase and Decrease Algorithms for Congestion Avoidance in Computer Networks, Computer Networks and ISDN Systems, vol. 17, pp. 1-14, 1998

[15] Stallings W. 2004. High-Speed Networks \& Internets Performance and Quality of Service, 2nd edition, Pearson Education, 2004

[16] Dmitri L., Hayder R. 2002. Increase-Decrease Congestion Control for Real-Time Streaming: Scalability, INFOCOM 2002. Proceedings of Twenty-First Annual Joint Conference of the IEEE Computer and Communications Societies.. IEEE Issue Volume 2, pp. 525-534, 2002.
[17] Jasem H. N., Zukarnain Z. A., Othman M., Subramaniam S. 2009. Fairness Of The TCP-Based New AIMD Congestion Control Algorithm, Journal of Theoretical and Applied Information Technology, 2009, pp. 568-576

[18] Jung H., Kim S-gyu, Yeom H. Y., et al. 2011. Adaptive Delay-based Congestion Control for High BandwidthDelay Product Networks, IEEE INFOCOM 2011, pp. 2885-2893

[19] Le L., Aikat J., Jeffay K., Smith F. D. 2007. The effects of active queue management on web performance, SIGCOMM, Karlsruhe, Germany, 2007, Vol. 15, Issue 6

[20] Jasem H N, Zukarnain Z.A., Othman M, Subramaniam S. 2009. The New AIMD Congestion Control Algorithm, World Academy of Science, Engineering and Technology 502009

[21] Braden B., Clark D., Crowcroft J., Davie B., Deering S., Estrin D., Floyd S., Jacobson V., Minshall G., Partridge C., Peterson L., Ramakrishnan K., Shenker S., Wroclawski J., and Zhang L. 1998. Recommendations on Queue Management and Congestion Avoidance in the Internet, Internet Engineering Task Force, RFC 2309, April 1998, URL: http://tools.ietf.org/html/rfc2309

[22] Floyd S. and Jacobson V. 1993. Random Early Detection Gateways for Congestion Avoidance, IEEE/ACM Transactions on Networking, vol. 1, no. 4, Aug. 1993

[23] Bolot M, Diot J, Lyles C, INRIA B. 1999. Reasons not to deploy RED, IWQoS '99. Seventh International Workshop on Quality of Service pp. 260-262, 1999.

[24] Alam M. S., Morshed M. N., SK Islam S., Azam M. M 2011. An Experimental Analysis of Random Early Discard RED) Queue for Congestion Control, International Journal of Computer Applications 0975 - 8887) Volume 15- No.2, February 2011

[25] Barrera I. D., Arce G. R., Bohacek S. 2011. Statistical approach for congestion control in gateway routers, A.Sprintson, Intl. journal of Computer and Telecomm Networking, Vol. 55, Issue 3, Feb 2011

[26] Le L. , Aikat J., Jeffay K., Smith F. D. 2007. The effects of active queue management on web performance, SIGCOMM, Karlsruhe, Germany, 2007, Vol. 15, Issue 6

[27] Ramakrishnan K. K., and Jain R. 1990. A Binary Feedback Scheme for Congestion Avoidance in Computer Networks, ACM Transactions on Computer Systems, V.8, N.2, pp. 152-181, 1990.

[28] Dorgham Sisalem, Henning Schulzrinne 1996. Congestion Control in TCP: Performance of Binary Congestion Notification Enhanced TCP Compared to Reno and Tahoe TCP, 1996. Proceedings of International Conference on Network Protocols, pp. $268-275$

[29] Sisalem D, Schulzrinne H. 1996. Congestion Control in TCP: Performance of Binary Congestion Notification Enhanced TCP Compared to Reno and Tahoe TCP, Network Protocols, 1996. Proceedings. IEEE, pp. 268-275

[30] Tang A, Jiantao Wang, Steven H. Low 2004 Understanding CHOKe: Throughput and Spatial Characteristics, IEEE/ACM Trans. Network, Vol. 12, Issue 4. pp. 694- 707, 2004.

[31] Tang A., Jiantao Wang, Steven H. Low 2004 Understanding CHOKe: Throughput and Spatial 
Characteristics, IEEE/ACM Trans. Network, Vol. 12, Issue 4. pp. 694- 707, 2004.

[32] Huang X., Sun G., Jing J., Zhang Z. 2009. An Approximating Fair Congestion Control Scheme at Core Network, First International Workshop on Education Technology and Computer Science, Vol. 01,IEEE 2009.

[33] Mathis M. and Mahdavi J. 1996. Forward Acknowledgement: Refining TCP Congestion Control, in Proc. ACM SIGCOMM, August 1996, pp.281-291.

[34] Shorten R. N., Leith D. J. 2007. On queue provisioning, network efficiency and the Transmission Control Protocol, IEEE/ACM Trans. On Networking, Aug 2007, Vol. 15, Issue:4, pp. 866-877

[35] Nyirenda C. N., Dong F., Hirota K. 2009. Reverse Traffic Reduction in the Fuzzy Logic based Dual Explicit Congestion Notification Mechanism in Satellite TCP/IP Networks, 4th Intl Symposium on Computational Intelligence and Intelligent Informatics, Oct 2009, pp. 4550 .

[36] Shakya S., Sainju A. 2011. ECN Congestion Control Mechanism in IP Networks 2011. Journal of the Institute of Engineering, Vol. 8, No. 1, pp. 12-24, ISSN: 1810-3383, 2010/2011

[37] Sharma A. R., Akkaya K. 2008. Real-time Routing for Mobile Sensor/Actor Networks, IEEE, Oct 2008, pp. 821828.

[38] Skoutas D. N., Rouskas A. N. 2010. Scheduling With QoS Provisioning in mobile broadband Wireless Systems, Europian wireless Conference, IEEE, Apr 2010, pp.422428.

[39] Shakkottai S. and Srikant R. 2002. Scheduling real-time traffic with deadlines over a wireless channel, Springer Wireless Networks, vol. 8, no. 1, pp. 13-26.

[40] Yang J. W., Cheng H-C, Fu L. C. 2008. Multiobjective Lot Scheduling and Dynamic OHT Routing in a $300-\mathrm{mm}$ Wafer Fab, 2008 IEEE International Conference on Systems, Man and Cybernetics SMC 2008. pp. 1608-1613

[41] Ramakrishnan K. K. and Raj Jain 1988. An Explicit Binary Feedback Scheme for Congestion Avoidance in Computer Networks with a Connection-less Network Layer, Proc. ACM SIGCOMM'88,Stanford, CA, August 1988, Vol. 8, Issue: 2, pp. 303-313

[42] Ramakrishnan K. K., Dah-Ming Chiu and Raj Jain1987. Congestion Avoidance in Computer Networks with a Connectionless Network Layer. Part IV: A Selective Binary Feedback Scheme for General Topologies, Digital Equipment Corporation, Technical Report TR-510, August 1987, pp. 47.

[43] Handley et al. 2003. TCP friendly Rate ControlTFRC):Protocol Specification, Network Working Group, Copyright C) The Internet Society, Jan 2003, pp-9

[44] Mo Jung, Nicolaos B. Karayiannis, and Steven Pei 2006. Improving TCP-Friendly Rate Control in Wired and Wireless Networks By a Scheme Based on Wireless Signal Strength, ICNS '06 Proc. Of Intl. Conference on Networking and Services, IEEE Society, 2006

[45] Gurtov A., Korhonen J. 2004. Measurement and Analysis of TCP-Friendly Rate Control for Vertical Handovers,
ACM Mobile Computing and Comm., July 2004, pp. 7387.

[46] Song L., Hu S., Mao P. et al. 2010. Performance Analysis of Explicit Control Protocol XCP. IET $3^{\text {rd }}$ Intl. Conference on Wireless, Mobile and MM Networks ICWMN 2010. pp. 40-43

[47] Low S. H., Andrew L. L. H., Wydrowski B P. 2005. Understanding XCP: Equilibrium and fairness, Appears in Proc. of IEEE Infocom, April 2005, Vol.2, pp. 1025-1036.

[48] Zhou H., Dai G., Ye F., Zhang H. 2009. Design of Controller for XCP Router Achieving Efficient and Fair Bandwidth Allocation, ISECS International Colloquium on Computing, Communication, Control, and Management, Aug 2009, pp. 282-285.

[49] Chowdhury I. S., Lahiry J., Hasan S. F. 2009. Performance Analysis of Datagram Congestion Control Protocol DCCP. Proceedings of 2009 12th International Conference on Computer and Information Technology ICCIT 2009. Dhaka, Bangladesh, pp. 21-23 December, 2009.

[50] Liu Y. M., Jiang, X. H. et al; 2009. Improved DCCP Congestion Control for Wireless Sensor Networks, IEEE/ACIS International Conference on Computer and Information Science, ICIS 2009, p. 194-198

[51] Min L. Y., Hua J. X., Hong N. X. 2009. Fairness for Extend DCCP Congestion Control in Wireless Sensor Networks, Chinese Control and Decision Conference, IEEE, CCDC '09, pp. 4732-4737

[52] Eddie Kohler, Sally Floyd 2003. Datagram Congestion Control Protocol DCCP) Overview, ICIR ,9 July 2003

[53] Dukkipati N, Ganjali Y and Shen R Z. 2005. Typical versus Worst Case Design in Networking, In Fourth Workshop on Hot Topics in Networks, College Park, Maryland, November 2005.

[54] Xu-Shan C., Rong W. X., Li-fei G. 2011. S-RCP:A Rate Control Protocol Algorithm Based on source control, Electronics, Communications and Control ICECC. International Conference, 9 Sep 2011, pp.2298-2301.

[55] Dukkipati N., McKeown N. 2006. Why Flow-Completion Time is the Right Metric for Congestion Control, In ACM SIGCOMM Computer Communication Review, Volume 36, Issue 1, January 2006.

[56] Gibb G., Mckeown N., Zhu J. 2007. Building a RCP Rate Control Protocol) Test Network, NSF, High-Performance Interconnects, 2007.15th Annual IEEE Symposium, 4 Sep 2007

[57] Dukkipati N., Kobayashi M., Zhang-Shen R., and McKeown N. 2005. Processor Sharing Flows in the Internet, Proceedings of International Workshop on Quality of Service IWQoS'05. Passau, Germany, June 2005.

[58] Lee J. H., Jung I. B. 2010. Adaptive-Compression Based Congestion Control Technique for Wireless Sensor Networks, Sensors 2010, Vol. no. 4 pp. 2919-2945, 29 March 2010.

[59] Ryu J., Bhargava V., Paine N. and Shakkottai S. 2010. Back-pressure routing and rate control for ICNs, in Proc MOBICOM, Sep 2010, pp.365-376. 
[60] Eryilmaz A. and Srikant R. 2006. Joint congestion control, routing, and MAC for stability and fairness in wireless networks, IEEE JSAC, Vol. 24, pp. 1514-1524, 2006.

[61] Rangwala S., Gummadi R., Govindan R., and Psounis K. 2006. Interference aware fair rate control in wireless sensor networks, in Proc. SIGCOMM, Pisa, Italy, Sep 2006, pp. 63-74.

[62] Payek J., Govindan R. 2010. RCRT: Rate-controlled reliable transport protocol for wireless sensor networks, Journal ACM Transactions on Sensor Networks TOSN), Vol. 7, Issue 3, September 2010

[63] Brahma S., Chatterjee M., Kwiat K. 2010. Congestion Control and Fairness in Wireless Sensor Networks, $8^{\text {th }}$ Intl. Conference On Pervasive Computing and Comm. Workshop PERCOM. IEEE 2010 pp. 413-418.

[64] Cheng TE., Bajcsy R. 2004. Congestion Control and Fairness for Many-to-One Routing in Sensor Networks. In Proc. Of $2^{\text {nd }}$ Intl. Conference On Embedded Networked Sensor Systems, SenSys 2004.

[65] Ahmad S., Mustafa A., Ahmad B., Bano A., Al Hosam 2009. Comparative Study of Congestion Control Techniques In High Speed Networks, IJCSIS, Vol.6, No. 2, 2009.

[66] Awan I., Ahmad S., Ahmad B. 2006. Performance analysis of multimedia based web traffic with QoS constraints, Journal of Computer and System Sciences 74 2008. pp. 232-242, 2006.
[67] Yerima S. Y., Al-Begain K. 2010. Novel Radio Link Buffer Management Schemes for End-User Multi-class Traffic in High Speed Packet Access Networks, Springer Science+Business Media, LLC. 2010, Vol. 61 No. 2, pp. 349-382

[68] Xu W., Zhou Z., Pham D. T., Ji C., Yang M., and Liu Q. 2011. Hybrid congestion control for high-speed networks, J. Network and Computer Applications, 2011, pp.14161428 .

[69] Hoda, Hisamatsu H., Hiroshi Noborio 2011) , Design, Implementation And Evaluation of Congestion Control Mechanism For Video Streaming, International Journal of Computer Networks \& Communications IJCNC), Vol.3, No.3, May 2011.

[70] Shoukat I.A., Iftikhar M., Haseeb A. 2011. Indexing size approximation of www repository with leading information retrieval and web filtering robots, Int. J. of Advanced Research in Comp. Sc. Vol. 2, No. 3, pp. 71-75, May-June (2011).

[71] Shoukat I.A., Ansari W.S., Iftikhar M. et. al. 2012.Practical Assessment of Remotely Communicated End point Servers of Leading Web Crawlers, IEEE Joint Conf. on Comp. Sc. and Software Eng. May 30 - June 01, IEEE (2012) pp. 219-224. 